\title{
Fatores potencializadores e limitadores na identificação e manutenção do potencial
}

\section{doador de órgãos}

Potentiating and limiting factors in the identification and maintenance of potential organ donors

Factores potenciadores y limitantes en la identificación y mantenimiento de posibles donantes de órganos

Cíntia Maria Lovato Flores

ORCID: https://orcid.org/0000-0001-6459-6454 Universidade Federal de Santa Maria, Brasil E-mail: florescintia1963@gmail.com

Rosângela Marion da Silva

ORCID: https://orcid.org/0000-0003-3978-9654 Universidade federal de Santa Maria, Brasil E-mail:cucasma@terra.com.br

Alexa Pupiara Flores Coelho Centenaro ORCID: https://orcid.org/0000-0002-9117-5847 Universidade Federal de Santa Maria, Brasil E-mail: alexa.coelho@ufsm.br

Silviamar Camponogara ORCID: https://orcid.org/0000-0001-9342-3683 Universidade Federal de Santa Maria, Brasil E-mail: silviaufsm@yahoo.com.br

Claudia Zamberlan

ORCID: https://orcid.org/0000-0003-1898-328X Universidade Franciscana, Brasil E-mail: czamberlab23@gmail.com

Juliana Tamiozzo

ORCID: https://orcid.org/0000-0003-3586-379X

Universidade Federal de Santa Maria, Brasil E-mail: julianatamiozzo4@gmail.com

\begin{abstract}
Resumo
Objetivo: analisar a produção do conhecimento, no contexto brasileiro, sobre as evidências relacionadas aos fatores potencializadores e limitadores reconhecidos pelos profissionais de saúde acerca da identificação e manutenção do potencial doador de órgãos e tecidos em morte encefálica e identificar propostas de intervenções para melhoria deste processo. Metodologia: Trata-se de uma revisão integrativa de literatura, desenvolvida nas bases de dados LILACS, BDENF e na biblioteca SCIELO, durante os meses de abril a junho de 2020. Resultados: O corpus da revisão constituiuse de 35 artigos, e após análise, identificou-se como fatores potencializadores a competência técnico-científica dos profissionais, a integração da equipe multiprofissional, conscientização, mobilização e sensibilização das equipes para o cuidado. Como fatores limitadores foi evidenciado a insegurança, inexperiência, o desconhecimento, o despreparo técnico e emocional, ausência de protocolos ou guidelines, as deficiências de recursos físicos, materiais, tecnológicos e humanos, a falta de maturidade profissional, pessoal e emocional, e a falta de uma cultura de doação. A educação permanente foi definida como a principal estratégia de intervenção para otimizar o processo. Conclusão: A importância do conhecimento que os profissionais têm sobre o assunto, o trabalho em equipe e a compreensão do significado de sua participação neste processo, assim como estratégias de educação permanente em saúde no ambiente hospitalar são necessários para aprimorar a qualidade da assistência prestada ao potencial doador de órgãos em morte encefálica.
\end{abstract}

Palavras-chave: Morte encefálica; Obtenção de tecidos e órgãos; Pessoal de saúde.

\begin{abstract}
Objective: to analyze the production of knowledge, in the Brazilian context, on the evidence related to the potentiating and limiting factors recognized by health professionals about the identification and maintenance of the potential donor of organs and tissues in brain death and to identify proposals for interventions to improve this process. Methodology: This is an integrative literature review, developed in the LILACS, BDENF and SCIELO library databases, from April to June 2020. Results: The review corpus consisted of 35 articles, and after analysis, the technical-scientific competence of the professionals, the integration of the multiprofessional team, awareness, mobilization and sensitization of the teams for care were identified as potentiating factors. As limiting factors, insecurity, inexperience, lack of knowledge, technical and emotional unpreparedness, absence of protocols or guidelines, deficiencies in physical, material,
\end{abstract}


technological and human resources, lack of professional, personal and emotional maturity, and lack of a giving culture. Permanent education was defined as the main intervention strategy to optimize the process. Conclusion: The importance of knowledge that professionals have on the subject, teamwork and understanding of the meaning of their participation in this process, as well as permanent health education strategies in the hospital environment are necessary to improve the quality of care provided to the patient. potential brain-dead organ donor.

Keywords: Brain death; Tissue and organ Procurement; Health personnel.

\section{Resumen}

Objetivo: analizar la producción de conocimiento, en el contexto brasileño, sobre las evidencias relacionadas con los factores potenciadores y limitantes reconocidos por los profesionales de la salud sobre la identificación y manutención del potencial donante de órganos y tejidos en muerte encefálica e identificar propuestas de intervención para mejora de este proceso. Metodología: Se trata de una revisión integrativa de la literatura, desarrollada en las bases de datos de las bibliotecas LILACS, BDENF y SCIELO, de abril a junio de 2020. Resultados: El corpus de revisión estuvo constituido por 35 artículos, y tras el análisis se valoró la competencia técnico-científica de los profesionales, se identificaron como factores potenciadores la integración del equipo multiprofesional, la concientización, movilización y sensibilización de los equipos para el cuidado. Como limitantes, la inseguridad, la inexperiencia, la falta de conocimientos, la falta de preparación técnica y emocional, la ausencia de protocolos o lineamientos, las deficiencias en los recursos físicos, materiales, tecnológicos y humanos, la falta de madurez profesional, personal y emocional, y la falta de una cultura generosa. La educación permanente se definió como la principal estrategia de intervención para optimizar el proceso. Conclusión: La importancia del conocimiento que los profesionales tengan sobre el tema, el trabajo en equipo y la comprensión del significado de su participación en este proceso, así como estrategias de educación permanente en salud en el ámbito hospitalario son necesarias para mejorar la calidad de la atención brindada al paciente. posible donante de órganos en muerte cerebral.

Palabras clave: Muerte encefálica; Obtención de tejidos y órganos; Personal de salud.

\section{Introdução}

A realidade brasileira, como de muitos países, é marcada pela dificuldade na obtenção de órgãos e tecidos para a realização dos transplantes. Para muitos pacientes portadores de algumas doenças terminais, o transplante de órgãos representa a última alternativa de tratamento, sendo preocupante a progressiva desproporção entre a necessidade por transplantes de órgãos e o baixo índice de transplantes realizados (Westphal et al., 2016).

No Brasil, o maior número de transplantes realizados é oriundo de doadores falecidos. Dados nacionais indicam que o número de transplantes realizados em 2019 obteve a taxa de 44 por milhão de população (pmp), sendo 38 pmp resultantes de doadores falecidos e a taxa de notificações de óbitos em morte encefálica foi de 54,7 pmp, ficando abaixo do piso nacional estimado que é de 70 pmp (Associação Brasileira de Transplantes de Órgãos [ABTO], 2019).

Morte encefálica (ME) é definida pela perda total das funções encefálicas, de caráter irreversível, com necessidade de uma causa neurológica conhecida que justifique o evento, se caracterizando pela cessação das atividades corticais e do tronco encefálico, o que equivale a morte da pessoa (Conselho Federal de Medicina [CFM], 2017). O paciente em ME é mantido com as funções dos órgãos preservadas temporariamente por meio de suporte da função cardiorrespiratória e, quando não há contraindicações, é considerado um potencial doador (PD). O diagnóstico de morte encefálica é de notificação compulsória às centrais de notificação, captação e distribuição de órgãos das unidades federadas onde tal fato ocorrer (Brasil, 1997).

O diagnóstico da morte encefálica é indispensável para que ocorra o processo de doação de órgãos, e também importante em pacientes não-doadores, pois torna-se indiscutível a sua realização devido a possibilidade de suspensão de procedimentos de suporte terapêutico, do ponto de vista ético e legal (CFM, 2007). Além disso, o diagnóstico proporciona a família do paciente, o direito de conhecer a situação real de seu familiar (Secretaria de Estado da Saúde do Paraná, 2018).

Estatísticas divulgadas por órgão competente (ABTO, 2018) revelam subnotificação dessas mortes e retardo no tempo para abertura do protocolo de M.E., o que leva a inviabilidade de sucesso no transplante. Muitas das dificuldades na oferta de órgãos para transplantes podem estar relacionadas a problemas na identificação da morte encefálica e na manutenção clínica do doador falecido. Neste raciocínio, considera-se imprescindível a sistematização do atendimento ao potencial doador de múltiplos órgãos (Westphal et al., 2016). No entanto, por ser tema considerado novo, ainda existem muitas dúvidas para os profissionais 
da saúde. Isto pode ser justificado pelo fato de que ainda no Brasil há uma minoria de instituições de ensino que abordam a morte encefálica e os transplantes como tema a ser discutido e trabalhado na formação, o que resulta em déficits posteriores desses profissionais no cuidado a estes pacientes (Costa et al., 2016).

Assim, o desenvolvimento de uma revisão integrativa com o intuito de aprofundar os conhecimentos relacionados à temática pode contribuir para aprimorar a atenção fornecida, uma vez que oferecerá subsídios para práticas mais efetivas e seguras no processo de trabalho. Dessa forma a revisão foi conduzida pela seguinte questão de pesquisa: Quais as evidências na produção científica acerca de fatores potencializadores e limitadores segundo os profissionais de saúde, no processo de identificação e manutenção do potencial doador de órgãos e tecidos em morte encefálica no contexto Brasileiro? Quais intervenções podem ser realizadas para a melhoria? O estudo teve como objetivo analisar a produção do conhecimento, no contexto brasileiro, sobre as evidências relacionadas aos fatores potencializadores e limitadores reconhecidos pelos profissionais de saúde, acerca da identificação e manutenção do potencial doador de órgãos e tecidos em morte encefálica e identificar propostas de intervenções para melhoraria deste processo.

\section{Metodologia}

Trata-se de uma revisão integrativa, realizada com a intenção de reunir, organizar e avaliar criticamente a produção científica, possibilitando a apresentação de evidências científicas de determinado assunto. A revisão seguiu os passos de planejamento e execução composto por formulação da pergunta de acordo com a estratégia PICo, amostragem com a seleção dos estudos que constituíram o corpus da revisão, extração dos dados dos estudos primários que respondiam a pergunta da revisão, análise crítica dos estudos com classificação de força de evidência, análise e síntese dos resultados da revisão e síntese do conhecimento (Paula et al., 2016).

A busca online foi realizada nos meses de abril a junho de 2020, por acesso ao Portal da Biblioteca Virtual de Saúde (BVS), e selecionada as bases de dados da Literatura Latino-Americana em Ciências da Saúde (LILACS) e Base de Dados em Enfermagem (BDENF); e na biblioteca Scientific Electronic Library Online (SciELO), utilizando as estratégias direcionadas para atender a pergunta estruturada na metodologia PICo (População $(\mathrm{P})=$ profissionais de saúde, Interesse $(\mathrm{I})=$ processo de identificação e manutenção do potencial doador de órgãos em morte encefálica, Contexto (Co) = brasileiro).

Os descritores foram selecionados segundo a classificação dos Descritores em Ciências da Saúde (DeCS): morte encefálica, obtenção de órgãos e tecidos, doação de órgãos, e classificação do Medical Subject Headings (MeSH): "brain death", Tissue and Organ Procurement. Estabeleceu-se recorte temporal a partir do ano de 2000. Os estudos nas bases de dados foram selecionados por diferentes estratégias para a BVS: "morte encefálica" AND ("doação de órgãos" OR "obtenção de tecidos e órgãos" OR "doador de órgãos" OR "doadores de órgãos") AND la:("en" OR "es" OR "pt")) AND (year_cluster: [2000 TO 2020]) e SCIELO: "brain death" AND "organ donation" OR "obtaining organs and tissues for donation" AND type:("researcharticle").

A seleção das produções foi desenvolvida de modo duplo independente, com vistas a eliminar a possibilidade de vieses nessa etapa. Para os critérios de inclusão foram adotados artigos com texto completo de pesquisas primárias sobre a temática, realizadas no contexto brasileiro, na língua portuguesa, inglesa ou espanhola, que respondessem a pergunta de revisão e publicados a partir do ano 2000, marco em relação a criação das Comissões Intra Hospitalares de Doação de Órgãos e Tecidos para Transplante (CIHDOTTs). A inclusão de estudos realizados no cenário brasileiro justifica-se pelo diagnóstico de morte encefálica e o processo de doação de órgãos serem regidos por leis e resoluções nacionais, e, portanto, podem variar de um país para outro. Para as produções que se encontravam em duplicidade, salienta-se que as mesmas foram consideradas apenas uma vez. 
Após leitura do título e resumo dos estudos e aplicação dos critérios de inclusão e exclusão, foram excluídos os estudos que não atendiam aos critérios de elegibilidade. Assim, foram selecionados 59 artigos que após a leitura na íntegra resultou no corpus da revisão constituído por 35 referências. Na Figura 1 é apresentado o fluxograma da seleção das produções.

Figura 1 - Fluxograma de seleção das produções, LILACS/BDENF/SCIELO.
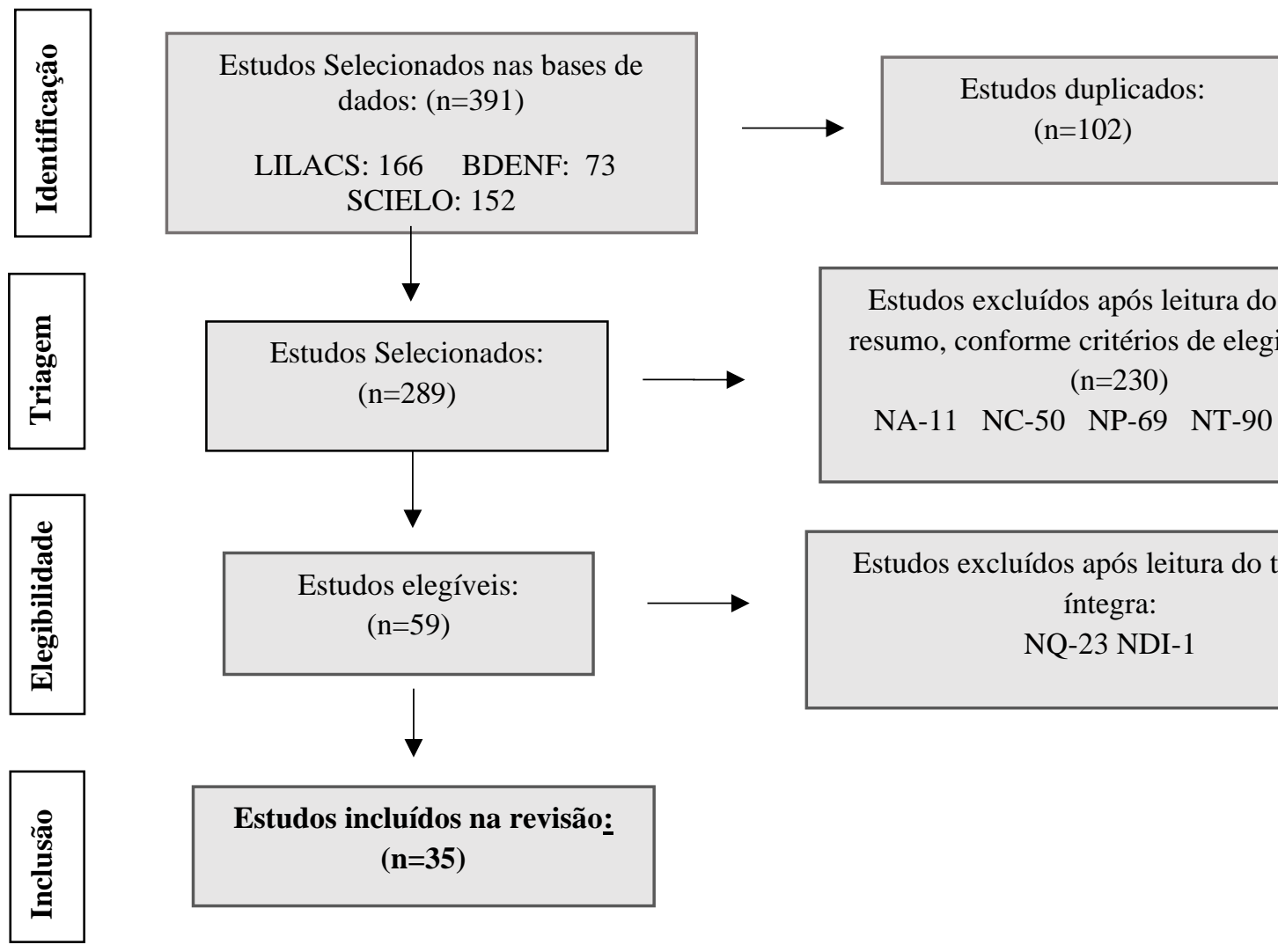

Estudos excluídos após leitura do título e resumo, conforme critérios de elegibilidade: $(n=230)$

NA-11 NC-50 NP-69 NT-90 NQ-10

Estudos excluídos após leitura do texto na íntegra:

NQ-23 NDI-1

Legenda: NA- não é artigo NC-não é do contexto NP- não é pesquisa original NT- não é da temática NQ- não responde à questão de pesquisa NDI: não disponível na íntegra questão de pesquisa. Fonte: Autores.

Para a extração dos dados, elaborou-se um quadro sinóptico composto pelas seguintes informações: identificação; título, autor, revista e ano; objetivos, delineamento do estudo/população; principais resultados e nível de evidência dos artigos. Quanto aos aspectos éticos, ressalta-se que foram respeitados os princípios de autoria e as citações dos autores das publicações que constituíram a amostra.

\section{Resultados}

As produções incluídas $(n=35)$ se caracterizaram por maioria com delineamento qualitativo $(n=22)$. Houve predomínio de estudos realizados na região Nordeste $(n=14)$. A áreas de conhecimento que se destacou foi a Enfermagem ( $n=26)$. Quanto à distribuição temporal, o arranjo quinquenal apontou a crescente publicação de estudos relacionados à temática de investigação entre os anos 2016 e $2019(n=17)$.

A avaliação crítica dos artigos segundo o sistema de classificação de força de evidências de Melnyk e Fineout-Overholt (Paula, C. C., Padoin, S. M. M., \& Galvão. C. M., 2016) mostrou que o maior número de estudos primários possuía sua questão clínica direcionada para o significado ou experiência $(n=22)$, com a maioria dos níveis de evidência classificados em 4 ( $n=26)$, conforme apresentado na Tabela 1 . 
Research, Society and Development, v. 11, n. 3, e42011326676, 2022

(CC BY 4.0) | ISSN 2525-3409 | DOI: http://dx.doi.org/10.33448/rsd-v11i3.26676

Tabela 1 - Caracterização das produções analisadas, LILACS/BDEnf/SciELO.

\begin{tabular}{|c|c|c|}
\hline Variáveis & $\mathbf{n}$ & $\%$ \\
\hline \multicolumn{3}{|l|}{ Abordagem do estudo } \\
\hline Qualitativo & 22 & 62,8 \\
\hline Quantitativo & 11 & 32,4 \\
\hline Quanti-Qualitativo & 2 & 5,7 \\
\hline \multicolumn{3}{|l|}{ Tipo do estudo } \\
\hline Exploratório Descritivo & 12 & 34,3 \\
\hline Teoria Fundamentada em Dados & 4 & 11,4 \\
\hline Descritivo & 4 & 11,4 \\
\hline Descritivo Transversal & 3 & 8,6 \\
\hline Etnografia & 2 & 5,7 \\
\hline Fenomenologia & 2 & 5,7 \\
\hline Discurso do Sujeito Coletivo & 1 & 2,9 \\
\hline Exploratória & 1 & 2,9 \\
\hline Análise Bardin & 1 & 2,9 \\
\hline Comparativo Exploratório & 1 & 2,9 \\
\hline Avaliativo Descritivo & 1 & 2,9 \\
\hline Transversal & 1 & 2,9 \\
\hline Sem descrição do tipo & 2 & 5,7 \\
\hline \multicolumn{3}{|l|}{ Procedência } \\
\hline Nordeste & 14 & 40 \\
\hline Sudeste & 8 & 22,9 \\
\hline Sul & 8 & 22,9 \\
\hline Centro-Oeste & 4 & 11,4 \\
\hline Norte & 1 & 2,9 \\
\hline \multicolumn{3}{|l|}{ Área do Conhecimento } \\
\hline Enfermagem & 26 & 74,3 \\
\hline Medicina & 6 & 17,1 \\
\hline Outra & 3 & 8,6 \\
\hline \multicolumn{3}{|l|}{ Distribuição Temporal } \\
\hline $2000-2005$ & 0 & 0,0 \\
\hline $2006-2010$ & 6 & 17,1 \\
\hline 2011-2015 & 12 & 34,3 \\
\hline 2016-2019 & 17 & 8,6 \\
\hline \multicolumn{3}{|c|}{ Questão de Pesquisa (estudo primário) } \\
\hline Significado & 22 & 62,9 \\
\hline Prognóstico/etiologia & 13 & 37,1 \\
\hline \multicolumn{3}{|l|}{ Classificação da evidência } \\
\hline Quatro & 26 & 74,3 \\
\hline Dois & 9 & 25,7 \\
\hline
\end{tabular}

Fonte: Autores.

Após análise minuciosa dos resultados obtidos nos estudos foi possível estabelecer categorias para responder à questão de pesquisa desta revisão, considerando a similaridade entre eles. Foram elencadas três categorias relacionadas aos fatores potencializadores e limitadores na identificação e manutenção do potencial doador: conhecimento da equipe multiprofissional, assistência no diagnóstico e manejo do potencial doador, e vivência/significado para os profissionais. Os resultados das evidências encontradas estão dispostos no Quadro 1. 
Quadro 1 - Fatores potencializadores e limitadores na identificação e manutenção do potencial doador falecido de órgãos e tecidos.

\section{IDENTIFICAÇÃO E MANUTENÇÃO DO POSSÍVEL OU POTENCIAL DOADOR DE ÓRGÃOS E TECIDOS EM MORTE} ENCEFÁLICA

\begin{tabular}{|c|c|}
\hline Fatores Potencializadores & Fatores Limitadores \\
\hline
\end{tabular}

\section{- $\quad$ Relacionados ao conhecimento:}

1. Competências técnico-científicas para reconhecer o diagnóstico de ME e atuar no cuidado direto aos pacientes no processo de ME (Carvalho, Sousa, Veloso \& Ataide, 2019; Cesar et al., 2019; Alves et al., 2019; F.A.A Silva et al., 2018)

2. Capacitação e educação permanente em saúde para a assistência ao paciente em ME e manutenção dos potenciais doadores (Cesar et al., 2019; I. F. da Costa et al., 2017; Longuiniere et al., 2016; A. L. P. Magalhães et al., 2018; J. V. Magalhães, Veras \& Mendes, 2016)

3. Profissionais demonstram conhecimento e experiência sobre a ME (Carvalho et al., 2019; Chehuen et al., 2019; F. A. A. Silva et al., 2018)

4. Compreensão da equipe de saúde sobre a fisiopatologia da morte encefálica (Andrade, Brito, Lira, Fernandes \& Melo, 2018; Cesar et al., 2019; Guimarães, Barbosa, Batista \& Passos, 2012)

\section{- Relacionados a Assistência}

1. Trabalho multiprofissional e integração da equipe (I. F. da Costa et al., 2017; Araujo \& Massarollo, 2014; A. L. P. Magalhães et al., 2018; Alves et al., 2019; T. R. Silva et al., 2018)

2. A UTI como ambiente hospitalar adequado para prestar um cuidado seguro e de qualidade aos pacientes no processo de ME e potencial doador (Aredes, Firmo \& Giacomin, 2018; A. L. P. Magalhães et al., 2018; Alves et al., 2019; T. R. Silva et al., 2018)

3. Organização de práticas de cuidado para manter a estabilidade hemodinâmica do paciente potencial doador em M.E. (Andrade et al., 2018; A. L. P. Magalhães et al., 2018)

4. A atuação da CIHDOTT e da Central de transplantes próximas as equipes assistentes (Andrade et al., 2018; Longuiniere et al., 2016; A. L. P. Magalhães et al., 2018)

5. Suporte da legislação brasileira para a doação e transplantes de órgãos (A. L. P. Magalhães et al., 2018)

6. A busca ativa de paciente em ME 14 (A. L. P. Magalhães et al., 2018)

7. Conscientização das equipes da necessidade de um cuidado efetivo ao paciente em ME (T. R. B. Silva, Nogueira \& Sá, 2016)

\section{- Relacionados ao conhecimento:}

1. Insegurança e desconhecimento da equipe de saúde (Amorim, Avelar \& Brandão, 2010; N. Costa et al, 2018; Cesar et al., 2019; Doria et al., 2016; Freire et al., 2014; Guido, Linch, Andolhe, Conegatto \& Tonini, 2009; Moraes, Santos, Merighi \& Massarollo, 2014; Neves, Duarte \& Mattia, 2008; Schein et al., 2008; D. R. S. de Souza, Tostes \& Silva, 2019; S. S. Souza, Borenstein, Silva, Souza \& Carvalho, 2013)

2. A formação dos profissionais de saúde (A. L. P. Magalhães et al., 2018; F. A. A. Silva et al., 2018)

3.Ausência de treinamentos e capacitações (Amorim et al., 2010; A. L. P. Magalhães et al., 2018; F. A. A. Silva et al., 2018; T. R. Silva, Alves, Braz \& Carbogim, 2018)

\section{- Relacionados a Assistência}

1. Dificuldade dos profissionais na aplicação dos critérios clínicos e condução do diagnóstico de ME (Chehuen et al., 2019; Araujo \& Massarollo, 2014; J. V. Magalhães et al., 2016)

2. Despreparo técnico e emocional para lidar com a situação da ME (Carvalho et al., 2019; Cesar et al., 2019; Araujo \& Massarollo, 2014; Vesco, N. L et al, 2016; Freire et al., 2014; Freire, Mendonça, Pontes, Vasconcelos \& Torres, 2012; Guido et al., 2009; A. L. P. Magalhães et al., 2018; Moraes et al., 2014; T. R. Silva et al., 2018) 3. Ausência ou desuso de protocolos ou guidelines (Freire et al., 2014; T. R. Silva et al., 2018)

4. Incompreensão do processo para a manutenção dos órgãos viáveis, em situações em que o paciente se encontra em morte encefálica (Cappellaro et al., 2014; T. R. Silva et al., 2018) 5. Sobrecarga emocional e física da enfermagem (A. L. P. Magalhães et al., 2018; S. S. Souza et al., 2013a)

6. Dimensionamento de recursos humanos inadequado (I. F. Costa et al., 2017; A. L. P. Magalhães et al., 2018; Moraes et al., 2014)

7. Enfermeiros não estarem atuando exclusivamente para atividades da OPO ou CIHDOTT (I. F. Costa et al., 2017)

8. A logística do processo de doação (Cesar et al., 2019; A. L. P. Magalhães et al., 2018)

9. Deficiências de recursos físicos, materiais, tecnológicos e humanos para a assistência ao PD (Amorim et al., 2010; I. F. Costa et al., 2017; Freire et al., 2014, 2012; Meneses et al, 2010; Moraes et al., 2014; T. R. Silva et al., 2018)

10. Morosidade na abertura e realização dos procedimentos de confirmação do diagnóstico de morte encefálica (Freire et al., 2012; Meneses et al, 2010; Moraes et al., 2014) 


\section{- Relacionado a vivência /significado:}

1. Associando o cuidado ao paciente no processo de ME à possibilidade de salvar outras vidas (Cesar et al., 2019; Alves et al., 2019)

2. Motivação para cuidar e sensibilização para doação de órgãos dos profissionais de saúde (Andrade et al., 2018; Moraes, Neves, Dos Santos, Merighi \& Massarollo, 2015; A. L. P. Magalhães et al., 2018)

3. Mudança na postura do profissional e modificação na concepção do cuidado ao ser em ME (Pestana, Erdmann \& Sousa, 2012)

\section{- Relacionados a vivência /significado:}

1. A definição, a constatação e a comunicação da morte encefálica envolvem percepções ambivalentes dos profissionais e dos familiares (Aredes et al., 2018; Carvalho et al., 2019; Cesar et al., 2019)

2. Falta de maturidade profissional, pessoal e emocional (Cavalcante, Ramos, Araújo, Alves \& Braga, 2014; Cesar et al., 2019; Araujo \& Massarollo, 2014; Moraes et al., 2014)

3. Descrença no Transplante e ausência de cultura da doação de órgãos (ABTO, 2019; Lemes \& Bastos, 2007)

Fonte: Autores.

A organização das categorias possibilitou sintetizar ações que podem ser implementadas pelos serviços de saúde na perspectiva de alavancar o processo de doação de órgãos e tecidos, por meio de identificação precoce da morte encefálica e o manejo adequado do potencial doador pelos profissionais de saúde, influenciando na qualidade dos órgãos a serem transplantados, como demonstrado no Quadro 2.

Quadro 2 - Propostas de ações para melhorias no processo de doação de órgãos nos serviços de saúde.

\section{PRINCIPAIS PROPOSTAS}

Incorporação de disciplinas com abordagem do tema nos cursos de graduação da área de saúde (Cesar et al., 2019; Doria et al., 2016; Pestana et al., 2012; D. R. S. Souza et al., 2019; Vesco et al., 2016).

Abordagem do tema de forma extensiva nos cursos de especialização em terapia intensiva (D. R. S. Souza et al., 2019).

Abordagem educacional para profissionais de saúde (N. Costa et al, 2018; Cesar et al., 2019; Chehuen et al., 2019; Freire et al., 2012; J. V. Magalhães et al., 2016; T. R. B. Silva, Nogueira \& Sá, 2016; D. R. S. de Souza et al., 2019; Vesco et al., 2016).

Desenvolver educação permanente e implantação de guidelines, protocolos e outros produtos que possam auxiliar de maneira efetiva no gerenciamento e assistência ao potencial doador de órgãos (N. Costa et al, 2018; A. L. P. Magalhães et al., 2018; F. A. A. Silva et al., 2018).

Discutir e repensar a atuação da equipe de enfermagem e seus conceitos sobre a morte, a vida, a solidariedade, com o intuito de aprimorar o cuidado ao potencial doador e facilitar a sua atuação no processo de captação de órgãos (S. S. Souza et al., 2013b).

Os profissionais precisam de apoio para enfrentar melhor estas situações que fazem parte do cotidiano de seu trabalho (Cesar et al., 2019; S. S. Souza et al., 2013b).

Adoção de estratégias que promovam o conhecimento e a mudança no comportamento do profissional (Freire et al., 2014).

A capacitação das equipes médicas quanto à morte encefálica não deve se restringir à legitimação do óbito e às melhorias nos índices de doação, precisa estar associada às concepções dos indivíduos acerca dos significados de vida e de morte, no seu contexto sociocultural (Aredes et al., 2018).

Investir no conhecimento e no desenvolvimento de habilidades e competências dos profissionais, principalmente daqueles que atuam em unidades de urgência e emergência ou em terapias intensivas, para que possam identificar um potencial doador, colaborar na identificação de morte encefálica, realizar a notificação na central de transplantes e envidar esforços para manter o potencial doador (Longuiniere et al., 2016; Neves et al., 2008).

A sensibilização do profissional, a humanização e a necessidade de intensificação nas intervenções e cuidados ao paciente em ME (T. R. B. Silva, Nogueira \& Sá, 2016).

Qualificação com o propósito de contemplar as dimensões técnica e bioética do cuidado ao paciente potencial doador de órgãos e sua família, levando em consideração a complexidade do processo (Cavalcante et al., 2014).

Fonte: Autores.

\section{Discussão}

A análise dos estudos permitiu identificar as evidências acerca de fatores potencializadores e limitadores no processo de identificação e manutenção do potencial doador. Estas se relacionam ao conhecimento da equipe multiprofissional, assistência no diagnóstico e manejo do potencial doador e vivência/significado para a equipe. 


\subsection{Conhecimento da Equipe Multiprofissional}

O conhecimento científico da equipe multiprofissional é condição essencial para o desempenho adequado na identificação e manutenção do potencial doador de órgãos, e, portanto, considerado um fator potencializador para o cuidado. Para os enfermeiros, possuir competências técnico-científicas, desenvolver o raciocínio clínico e reconhecer o diagnóstico de ME permite atuar com segurança no cuidado direto aos pacientes neste processo, junto à equipe multiprofissional, e está estritamente relacionado à formação do profissional (Alves et al., 2019).

A educação permanente em saúde pode contribuir na melhoria do cuidado aos potenciais doadores e reduzir o tempo para realização de testes que determinem a ME (I. F. Costa et al., 2017). Estudo (J. V. Magalhães et al., 2016) que buscou avaliar o conhecimento dos médicos intensivistas sobre morte encefálica, concluiu que existe a necessidade de atualização profissional, visto que observou o desconhecimento sobre a definição de morte encefálica, desconsideração sobre os exames que devem ser realizados para o diagnóstico ser validado, e fragilidades no momento da execução do protocolo. As capacitações para qualificação profissional podem repercutir no aumento no número de doadores efetivos (Silva et al., 2018; T. R. Silva et al., 2018), constituindo-se um dos fatores que contribuíram para a aplicação dos critérios de morte encefálica, sobretudo após a mudança recente das normas brasileiras sobre morte encefálica (Chehuen et al., 2019).

Há clareza do papel da educação permanente em saúde e a necessidade das instituições de saúde em promover capacitações aos seus profissionais a respeito da ME e sobre o cuidado aos potenciais doadores, possibilitando espaço para a aquisição de novos conhecimentos e aprimoramento profissional (N. Costa et al, 2018). As atividades de educação e aperfeiçoamento devem ser contínuas para os profissionais de saúde, proporcionando condutas mais uniformes dos profissionais (Vesco et al., 2016). Com o desenvolvimento da educação permanente, criam-se espaços para a implantação de protocolos, guidelines, e outras tecnologias para subsidiar de maneira efetiva a assistência ao possível e potencial doador de órgãos (A. L. P. Magalhães et al., 2018).

A insegurança e o desconhecimento foram citados em vários estudos pelos profissionais de saúde como fatores limitadores na identificação e manutenção do potencial doador de órgãos (Amorim et al., 2010; N. Costa et al, 2018; Cesar et al., 2019; Doria et al., 2016; Freire et al., 2014; Guido et al., 2009; Moraes, Santos, Merighi \& Massarollo, 2014; Neves et al., 2008; S. S. Souza et al., 2013a; Schein et al., 2008; D. R. S. Souza et al., 2019). O conhecimento frágil da equipe multiprofissional acerca do manejo com o potencial doador afeta diretamente no desenvolvimento das etapas de identificação, diagnóstico de ME e manutenção do doador em condições adequadas para a preservação dos órgãos (Moraes et al., 2014), o que leva a depreender que a assistência prestada a estes pacientes deverá sofrer mudanças para o aperfeiçoamento (N. Costa et al, 2018).

Pesquisa (Freire et al., 2014) realizada com profissionais da equipe de enfermagem identificou como principais dificuldades na assistência ao potencial doador o despreparo da equipe e a falta de conhecimento, especialmente sobre a fisiopatologia da ME e o impacto da mesma sobre o organismo (Amorim et al., 2010).

Um estudo (F. A. A. Silva et al., 2018) identificou que a maioria dos profissionais de saúde intensivistas não realizaram treinamento para o manejo do potencial doador e a preservação adequada dos órgãos para o transplante, o que pode ter fragilizado a qualidade da assistência prestada por esses profissionais.

A abordagem educacional para profissionais de saúde está presente em muitas das conclusões dos estudos da revisão (T. R. B. Silva, Nogueira \& Sá, 2016; N. Costa et al, 2018; Cesar et al., 2019; Chehuen et al., 2019; Vesco et al., 2016; Freire et al., 2012; Schein et al., 2008; D. R. S. Souza et al., 2019), tornando-se crucial a sua implementação para a melhoria do processo. Porém, a falta de inclusão do tema na formação dos profissionais de saúde contribui para dificultar a assistência prestada ao potencial doador de órgãos (A. L. P. Magalhães et al., 2018), sendo sugerido a incorporação de disciplinas que abordem o tema nos cursos de formação da área de saúde (Cesar et al., 2019; Vesco et al., 2016; Doria et al., 2016; Pestana et al., 2012; D. R. S. 
de Souza et al., 2019). Também é adequada a discussão do tema de maneira abrangente nos cursos de especialização (D. R. S. de Souza et al., 2019).

\subsection{Assistência ao Potencial Doador}

Em relação a assistência ao potencial doador de órgãos em ME, foram identificados estudos que elencaram os fatores potencializadores do processo, entre eles a integração da equipe e o trabalho multiprofissional. Decorrente da complexidade do cuidado ao paciente em ME, este exige a interdependência das práticas de cuidado (A. L. P. Magalhães et al., 2018), sendo o trabalho multiprofissional reconhecido como importante facilitador da doação de órgãos e tecidos para transplantes (Alves et al., 2019).

Outra condição identificada foi a garantia do cuidado intensivo ao paciente/potencial doador. A UTI é o local que se diferencia no contexto hospitalar, com competência, para o cuidado aos pacientes no processo de ME, tornando-o seguro e de qualidade (Alves et al., 2019). Esta unidade é considerada como o local modelo para a realização da gerência do cuidado ao paciente em ME, por integrar, estrutura física, recursos materiais e número de pessoal adequado (T. R. Silva et al., 2018). A destinação de leito em unidade com profissionais capacitados propiciará a confirmação ou não da morte encefálica mais precocemente, e a viabilidade de pacientes doadores de órgãos (Aredes et al., 2018).

A necessidade de manutenção da estabilidade hemodinâmica do potencial doador de órgãos é propiciada pela organização de práticas do cuidado (A. L. P. Magalhães et al., 2018). Tanto o enfermeiro de terapia intensiva como o da Comissão Intra Hospitalar de Doação de Órgãos para Transplante (CIHDOTT) devem trabalhar no planejamento destas práticas de cuidado (A. L. P. Magalhães et al., 2018).

A atuação da CIHDOTT, da Organização de Procura de Órgãos (OPO) e da Central de Transplantes próximas as equipes assistentes são consideradas aspectos positivos no processo de identificação e manutenção do potencial doador de órgãos (Andrade et al., 2018; Longuiniere et al., 2016; A. L. P. Magalhães et al., 2018). Também contribuem como suporte, a legislação brasileira para a doação de órgãos e transplantes e a busca ativa de pacientes em morte encefálica (A. L. P. Magalhães et al., 2018).

A conscientização das equipes da necessidade de um cuidado efetivo a este paciente é elemento que contribui para uma melhor assistência ao paciente em ME. A incorporação para si da responsabilidade no processo da ME é explicada pela consciência destes profissionais da importância do cuidado ao potencial doador de órgãos (T. R. B. Silva, Nogueira \& Sá, 2016).

Entre os fatores limitadores enfrentados na assistência ao potencial doador de órgãos cita-se a dificuldade dos profissionais em realizar o diagnóstico de ME (Chehuen et al., 2019; Araujo \& Massarollo, 2014; J. V. Magalhães et al., 2016) e os conflitos acirrados na equipe multiprofissional decorrente da diversidade de opiniões sobre a conduta para a realização do protocolo de morte encefálica (Freire et al., 2014).

O despreparo técnico e emocional para lidar com a situação da ME das equipes é apontada por estudos (Carvalho et al., 2019; Cesar et al., 2019; Araujo \& Massarollo, 2014; Freire et al., 2014, 2012; A. L. P. Magalhães et al., 2018; Moraes et al., 2014; T. R. Silva et al., 2018; Vesco et al., 2016). A inaptidão relacionada a falta de equilíbrio profissional, pessoal e emocional pode conduzir ao afastamento do profissional do cuidado ao paciente em morte encefálica (Cavalcante et al., 2014; Cesar et al., 2019). Um dos empecilhos elencados pela equipe de enfermagem na assistência aos potenciais doadores é a falta de conhecimento associada ao despreparo da equipe (Freire et al., 2014). Assim como, a ausência ou o não uso de protocolos ou guidelines dificulta uma assistência uniforme e segura (Freire et al., 2014; T. R. Silva et al., 2018).

A incompreensão do processo para a manutenção dos órgãos viáveis é percebida pelo modo com que os profissionais de enfermagem vislumbram o paciente em ME, sem lhe atribuir sua real importância, muitas vezes relacionada a ausência de uma cultura de doação de órgãos (T. R. Silva et al., 2018). A compreensão da complexidade do processo para a manutenção dos 
órgãos em condições adequadas do paciente em morte encefálica pelos profissionais de saúde é impedida pelos dilemas éticos presentes (Cappellaro et al., 2014).

A sobrecarga emocional e física da enfermagem é marcante na atuação da equipe no atendimento do potencial doador. Estudo (S. S. Souza et al., 2013b) evidenciou o sofrimento da equipe de enfermagem durante a atuação junto ao potencial doador, a qual necessita buscar estratégias de enfrentamento para as situações de estresse, e, facilitar seu trabalho. É reconhecido pelos enfermeiros da UTI que o profissional se distancia do paciente por desconhecimento, despreparo psicológico e emocional em lidar com a situação (A. L. P. Magalhães et al., 2018).

A inadequação do dimensionamento de recursos humanos é atribuída como fator dificultador (I. F. Costa et al., 2017; A. L. P. Magalhães et al., 2018; Moraes et al., 2014). A carência de recursos humanos e materiais leva a equipe de saúde a não considerar o doador como uma prioridade, ficando em segundo plano na assistência (Moraes et al., 2014), e que somado a inexistência de profissionais exclusivos para as atividades da OPO ou CIHDOTT podem fragilizar o processo de manutenção do potencial doador (I. F. Costa et al., 2017). As deficiências de recursos físicos, materiais, tecnológicos e humanos para a assistência ao PD (Amorim et al., 2010; I. F. Costa et al., 2017; Freire et al., 2014, 2012; Meneses et al., 2010; Moraes et al., 2014; T. R. Silva et al., 2018) se relacionam a pouca disponibilidade de equipamentos para o diagnóstico de ME, ao número reduzido de pessoal, a estrutura física deficiente, conduzindo ao stress da equipe, que se frustra por não assistir adequadamente ao potencial doador (T. R. Silva et al., 2018).

A logística do processo de doação também interfere no andamento da identificação e manutenção do potencial doador (A. L. P. Magalhães et al., 2018), visto que é um processo complexo e exige uma organização e interação entre os serviços. É mencionado também como um fato negativo, a morosidade na abertura e realização dos procedimentos confirmatórios para o diagnóstico de morte encefálica (Freire et al., 2012; Moraes et al., 2014).

Desta forma, algumas propostas se destacam, como a necessidade de apoio institucional para melhor enfrentamento das situações que envolvem a ME pelos profissionais que prestam assistência ao potencial doador e sua família (S. S. Souza et al., 2013). Investir não somente na capacitação de profissionais que atuam em Terapia Intensiva, mas nas Unidades de Urgência/Emergência, com estratégias que promovam o desenvolvimento de habilidades e competências na identificação precoce da morte encefálica e assistência ao potencial doador, empregando esforços para a sua manutenção (Neves et al., 2008) com o intuito de potencializar as etapas para a viabilidade dos transplantes (Longuiniere et al., 2016).

A mudança de postura do profissional está intrinsecamente ligada à promoção do conhecimento dos mesmos (Freire et al., 2014), contemplando não só o enfoque técnico, mas também os valores bioéticos que estão em questão, considerando a complexidade do cuidado ao ser em ME/potencial doador de órgãos e sua família (Cavalcante et al., 2014).

\subsection{Vivência/Significado para os Profissionais}

A falta de maturidade profissional, pessoal e emocional é encarado como um agravante (Cavalcante et al., 2014), pois o desconhecimento, a inexperiência e a resistência para a abertura do diagnóstico estão relacionadas a dificuldades por parte da equipe de saúde em não aceitação da morte encefálica como sendo a morte da pessoa, impedindo a sua comprovação (Carvalho et al., 2019; Moraes et al., 2014).

A ausência de cultura de doação de órgãos no país é um obstáculo identificado na literatura (T. R. Silva et al., 2018) quando relacionam a inexistência de uma cultura de doação de órgãos com a forma como o potencial doador é visto pela equipe multiprofissional, quando é assistido apenas como um paciente morto, sem atribuir-lhe a sua real importância, o que reflete na maneira como os profissionais de enfermagem dão contribuição para que este potencial doador possa vir a dar vida e sobrevida a outros pacientes que aguardam por tais órgãos. Assim, também o afastamento da equipe do paciente em morte encefálica é 
determinado pelas crenças e valores dessa subcultura (Cesar et al., 2019; Lemes \& Bastos, 2007), que pode justificar a falta de importância dada a este paciente (Freire et al., 2014).

Diante do exposto, o aspecto pessoal e emocional da equipe multiprofissional precisa ser valorizado, e a abertura para espaços de discussão sobre o tema sugere aos profissionais, repensar seus conceitos sobre a vida e a morte, a sua atuação e responsabilidade no processo de doação de órgãos (S. S. Souza et al., 2013b). A sensibilização do profissional e a humanização do processo são mencionadas como ações necessárias (T. R. B. Silva, Nogueira \& Sá, 2016).

Há de se ressaltar que muitos dos problemas da equipe que trabalha em unidades que internam pacientes com possível diagnóstico de ME está na compreensão do significado da ME por parte dos profissionais, e na importância de sua participação neste processo. Cabe elucidar a relevância da educação permanente, ficando a cargo das instituições, em todos os níveis, responsáveis pela doação de órgãos, estender este conhecimento ao maior número possível de profissionais, bem como a intensificação de campanhas junto à população para elucidar o tema. Há necessidade do trabalho com uma equipe coesa e multidisciplinar, no sentido de buscar alternativas e soluções para as adversidades de cada fase do processo, com o envolvimento de todos nessa ação.

Por fim destaca-se que muitos estudos desta revisão têm como população da pesquisa a equipe de enfermagem. E sendo os profissionais de enfermagem os que acompanham o potencial doador de órgãos com maior proximidade durante sua internação, desde a chegada ao hospital até o desfecho final, acredita-se que esta revisão possa fornecer subsídios para que, cada vez mais, seja proporcionado a estes e aos demais profissionais da saúde, condições para o agir e assistir ao potencial doador de órgãos na sua complexidade.

Desta forma, poderá ser proporcionado às pessoas que fazem parte da lista de espera por um órgão, maior possibilidade de realização do transplante com sucesso, o que significa maior qualidade de vida, ou a própria vida. Para a família do doador, este ato poderá significar muito na elaboração do luto, dando um sentido à perda do ente querido.

\section{Considerações Finais}

Ao analisar a produção do conhecimento, identificou-se vários fatores potencializadores para a identificação e manutenção do potencial doador de órgãos, entre eles a competência técnico-científica, a integração da equipe multiprofissional, conscientização, mobilização e sensibilização das equipes para o cuidado. Como fatores limitadores aparecem a insegurança, a inexperiência, o desconhecimento, o despreparo técnico e emocional, ausência de protocolos ou guidelines, as deficiências de recursos físicos, materiais, tecnológicos e humanos, a falta de maturidade profissional, pessoal e emocional, e a falta de uma cultura de doação, destacam-se entre os principais.

O que permite concluir que é imperiosa a educação dos profissionais de saúde, relativa ao tema, tanto a nível da graduação e em cursos de especialização, bem como treinamentos e capacitações ofertados pelas instituições de saúde responsáveis. Salienta-se a importância do apoio de dirigentes na implantação de guidelines, protocolos e outros serviços que venham a contribuir para melhoria do processo, como também prestar auxílio e assistência psicológica à equipe multiprofissional. Assim, sugere-se que novos estudos sejam realizados envolvendo a atuação dos profissionais de saúde na identificação da ME e manutenção do PD, dando ênfase para modelos e avaliação de estratégias implementadas nas equipes de trabalho.

\section{Referências}

Amorim, V. C. D., Avelar, T. A. B. A., \& Brandão, G. M. O. do N. (2010). A otimização da assistência de enfermagem ao paciente em morte encefálica: potencial doador de múltiplos órgãos. Rev. Enferm. UFPE $218-226$. http://www.ufpe.br/revistaenfermagem/index.php/revista/article/viewFile/726/463

Alves, M. P., Rodrigues, F. S., Cunha, K. S., Higashi, G. D. C., Nascimento, E. R. P., \& Erdmann, A. L.(2019). Processo de morte encefálica: significado para enfermeiros de uma unidade de terapia intensiva. Revista Baiana de Enfermagem33, . https://doi.org/10.18471/rbe.v33.28033 
Andrade, J. D. A. de, Brito, A. C. de, Lira, G. G., Fernandes, F. E. C. V., \& Melo, R. A. de. (2018). Vivências e estratégias de uma organização de procura de órgãos. Revista de Enfermagem UFPE on Line, 12(4), 857. https://doi.org/10.5205/1981-8963-v12i4a110257p857-864-2018

Araujó, M. N., \& Massarollo, M. C. K. B. (2014). Conflitos éticos vivenciados por enfermeiros no processo de doação de órgãos. Acta Paulista de Enfermagem, 27(3), 215-220. https://doi.org/10.1590/1982-0194201400037

Aredes, J. de S., Firmo, J. O. A., \& Giacomin, K. C. (2018). A morte que salva vidas: complexidades do cuidado médico ao paciente com suspeita de morte encefálica. Cadernos de Saúde Pública, 34(11). https://doi.org/10.1590/0102-311X00061718

Associação Brasileira de Transplantes de Órgãos (2019). Dimensionamento dos Transplantes no Brasil e em cada estado (2012-2019). Registro Brasileiro de Transplantes, XXV (4). http://www.abto.org.br/abtov03/Upload/file/RBT/2019/RBT-2019-leitura.pdf.

Associação Brasileira de Transplantes de Órgãos (2018). Dimensionamento dos Transplantes no Brasil e em cada estado (2011-2018). Registro Brasileiro de Transplantes, XXIV (4). http://www.abto.org.br/abtov03/Upload/file/RBT/2018/Lv_RBT-2018.pdf

Brasil (1997). Lei n 9434 de 4 de fevereiro de 1997. http://www.planalto.gov.br/ccivil_03/LEIS/L9434.htm

Cappellaro, J., Silveira, R. S. da, Lunardi, V. L., Corrêa, L. V. O., Sanchez, M. L., \& Saioron, I. (2014). Comissão Intra-Hospitalar de Doação de Órgãos e Tecidos para Transplante: questões éticas. Rev. RENE, 15(6), 949-956. http://www.revistarene.ufc.br/revista/index.php/revista/article/view/1813/pdf

Carvalho, N. de S., Sousa, J. de, Veloso, L. C., \& Ataide, karine de M. N. (2019). Atuação do enfermeiro no processo de doação e captação de órgãos em doadores elegíveis. Revista de Enfermagem Da UFPI, 8(1), 23-29. Retrieved from https://ojs.ufpi.br/index.php/reufpi/article/view/7289

Cavalcante, L. de P., Ramos, I. C., Araújo, M. Â. M., Alves, M. D. dos S., \& Braga, V. A. B. (2014). Cuidados de enfermagem ao paciente em morte encefálica e potencial doador de órgãos. Acta Paulista de Enfermagem, 27(6), 567-572. https://doi.org/10.1590/1982-0194201400092

Cesar, M. P., Camponogara, S., Da Cunha, Q. B., Pinno, C., Girardon-Perlini, N. M. O., \& Flores, C. L. (2019). Percepções e experiências de trabalhadores de enfermagem sobre o cuidado ao paciente em morte encefálica. Revista Baiana de Enfermagem33, . https://doi.org/10.18471/rbe.v33.33359

Chehuen Neto, J. A., Ferreira, R. E., Assad, I. M., Santos, I. A., Santos, J. L. C. T. Dos, Paula, L. C., \& Breder, S. D. (2019). Atualização dos critérios diagnósticos de morte encefálica: aplicação e capacitação dos médicos. Revista Brasileira de Terapia Intensiva, 31(3), 303-311. https://doi.org/10.5935/0103-507X.20190055

Conselho Federal de Medicina (2017). Resolução CFM nº 2173. Brasília: CFM. https://sistemas.cfm.org.br/normas/visualizar/resolucoes/BR/2017/217

Conselho Federal de Medicina (2007). Resolução CFM n ${ }^{\text {0 }}$ 1.826. http://www.portalmedico.org.br/resolucoes/cfm/2007/1826_2007.htm.

Costa, C. R., Costa, L. P., \& Aguiar, N. (2016). A enfermagem e o paciente em morte encefálica na UTI. Revista Bioética, 24(2), 368-373. https://doi.org/10.1590/1983-80422016242137

Costa, I. F., Mourão, J. J., Brito, M. C. C., Goyanna, N. F., Santos, T. C., \& Santos, S. S. (2017). Fragilidades na atenção ao potencial doador de órgãos: percepção de enfermeiros. Revista Bioética, 25(1), 130-137. https://doi.org/10.1590/1983-80422017251174

Costa, N., Oliveira, L., Santos, A., Leal, H., \& Sousa, T. (2018). Manejo dos pacientes em morte encefálica. Revista de Enfermagem UFPE on line, 12(4), 953961. doi:https://doi.org/10.5205/1981-8963-v12i4a110145p953-961-2018

Doria, D., Doria, D. L., Leite, P. M. G., Brito, F. P. G., Brito, G. M. G. de, Resende, G. G. S., \& Santos, F. L. L. S. M. (2016). Conhecimento do enfermeiro no processo de doação de órgãos. Enfermagem Em Foco, 6(1/4), 31-35. https://doi.org/10.21675/2357-707X.2015.v6.n1/4.573

Freire, I. L. S., Mendonça, A. E. O. de, Freitas, M. B. de, Melo, G. D. S. M., Costa, I. K. F., \& Torres, G. D. V. (2014). Conocimiento del equipo de enfermería sobre la muerte encefálica y la donación de órganos. Enfermería Global, 13(4), 179-207. https://doi.org/10.6018/eglobal.13.4.175961

Freire, I. L. S., Mendonça, A. E. O. de, Pontes, V. O. de, Vasconcelos, Q. L. D. A. Q., \& Torres, G. de V. (2012). Morte encefálica e cuidados na manutenção do potencial doador de órgãos e tecidos para transplante. Revista Eletrônica de Enfermagem, 14(4), 903-912. https://doi.org/10.5216/REE.V14I4.14598

Guido, L. de A., Linch, G. F. da C., Andolhe, R., Conegatto, C. C., \& Tonini, C. C. (2009). Stressors in the nursing care delivered to potential organ donors TT - Factores de estrés en la asistencia de enfermería al potencial donador de órganos TT - Estressores na assistência de enfermagem ao potencial doador de órgãos. Rev. Latinoam. Enferm, 17(6), 1023-1029. Retrieved from http://scielo.br/scielo.php?script=sci_arttext\&pid=S0104-11692009000600015

Guimarães, J. B., Barbosa, N. M., Batista, M. de A., \& Passos, X. S. (2012). Conhecimento dos enfermeiros sobre condutas na prevenção, manutenção e no controle da temperatura de potenciais doadores de órgãos. J. Health Sci. Inst, 30(4). Retrieved from http://www.unip.br/comunicacao/publicacoes/ics/edicoes/2012/04_out-dez/V30_n4_2012_p365a368.pdf

Lemes, M. M.D.D \& Bastos, M. A.R. (2007). Os cuidados de manutenção dos potenciais doadores de órgãos: estudo etnográfico sobre a vivência da equipe de enfermagem. Revista Latino Americana de Enfermagem, 15(5). Retrieved from www.eerp.usp.br/rlae

Longuiniere, A. C. F. D. La, Lobo, M. P., Leite, P. L., Barros, R. D. C. S., Souza, A. N., \& Vieira, S. N. S. (2016). Knowledge of critical care nurses about the process of brain death diagnosis. Revista Da Rede de Enfermagem Do Nordeste, 17(5), 691. https://doi.org/10.15253/2175-6783.2016000500015

Magalhães, A. L. P., Erdmann, A. L., Sousa, F. G. M. de, Lanzoni, G. M. de M., Silva, E. L. da, \& Mello, A. L. S. F. de. (2018). Significados do cuidado de enfermagem ao paciente em morte encefálica potencial doador. Revista Gaúcha de Enfermagem, 39(0), e20170274. https://doi.org/10.1590/19831447.2018.2017-0274

Magalhães, J. V., Veras, K. N., \& Mendes, C. M. de M. (2016). Avaliação do conhecimento de médicos intensivistas de Teresina sobre morte encefálica. Revista Bioética, 24(1), 156-164. https://doi.org/10.1590/1983-80422016241117

Meneses, E. A., Souza, M. F. B., Baruzzi, R. M., Machado Do Prado, M., \& Garrafa, V. (2010). Análise bioética do diagnóstico de morte encefálica e da doação de órgãos em hospital público de referência do Distrito Federal. Revista Bioética, 18(2), 397-412. Retrieved from 
https://revistabioetica.cfm.org.br/index.php/revista_bioetica/article/view/572

Moraes, E. L., Neves, F. F., Dos Santos, M. J., Merighi, M. A. B., \& Massarollo, M. C. K. B. (2015). Experiências e expectativas de enfermeiros no cuidado ao doador de órgãos e à sua família. Revista Da Escola de Enfermagem Da USP, 49(SpecialIssue2), 129-135. https://doi.org/10.1590/S0080-623420150000800018

Moraes, E. L. de, Santos, M. J. dos, Merighi, M. A. B., \& Massarollo, M. C. K. B. (2014a). Experience of nurses in the process of donation of organs and tissues for transplant. Revista Latino-Americana de Enfermagem, 22(2), 226-233. https://doi.org/10.1590/0104-1169.3276.2406

Neves, A. R. das, Duarte, E., \& Mattia, A. L. De. (2008). Notificação de morte encefálica em doação de órgâos. REME Rev. Min. Enferm, $213-218$.

Paula, C. C., Padoin, S. M. M., \& Galvão. C. M. (2016). Revisão integrativa como ferramenta para tomada de decisão na prática em saúde. In: Lacerda, M. R. \& Costenaro, R. G. S. (Orgs). Metodologias da Pesquisa para a enfermagem e Saúde: da teoria à prática (p.51-76). Porto Alegre: Moriá.

Pestana, A. L., Erdmann, A. L., \& Sousa, F. G. M. de. (2012). Emergindo a complexidade do cuidado de enfermagem ao ser em morte encefálica. Escola Anna Nery, 16(4), 734-740. https://doi.org/10.1590/S1414-81452012000400013

Schein, A. E., Carvalho, P. R. A., Rocha, T. S. da, Guedes, R. R., Moschetti, L., La Salvia, J. C., \& La Salvia, P. C. (2008). Avaliação do conhecimento de intensivistas sobre morte encefálica. Revista Brasileira de Terapia Intensiva, 20(2), 144-148. https://doi.org/10.1590/S0103-507X2008000200005

Secretaria de Estado da Saúde do Paraná (2018). Manual para notificação, diagnóstico de morte encefálica e manutenção do potencial doador de órgãos e tecidos $3^{\text {a }}$ ed. [Manual]. Curitiba: Sistema de http://coimplante.odo.br/Biblioteca/Publica\%C3\%A7\%C3\%B5es\%202019/Protocolo\%20de\%20Morte\%20Encef\%C3\%A1lica\%2003.09\%20novo.pdf.

Silva, F. A. A., Cunha, D. S. P., Lira, J. A. C., Ribeiro, J. F., Campelo, G. V. de S., \& Nunes, B. M. V. T. (2018). Morte encefálica e manutenção de órgãos: conhecimento dos profissionais intensivistas TT - Brain death and organ maintenance: knowledge of intensive care professionals. Rev. enferm. UFPE on line, 12(1), 51-58. Retrieved from https://periodicos.ufpe.br/revistas/revistaenfermagem/article/view/25130/25852

Silva, T. R. B., Nogueira, M. A., \& Sá, A. M. M. (2016). Conhecimento da equipe de enfermagem acerca dos cuidados com o potencial doador em morte encefálica. Rev. enferm. UFPI, 5(4), 24-30. Retrieved from http://www.ojs.ufpi.br/index.php/reufpi/article/view/5641

Silva, T. R., Alves, M. D. S., Braz, P. R., \& Carbogim, F. D. C. (2018). Comissão Intra-Hospitalar de Doação de Órgãos e Tecidos para Transplante: vivência dos enfermeiros. Revista Enfermagem UERJ, 26(0), e34120. https://doi.org/10.12957/reuerj.2018.34120

Souza, D. R. S., Tostes, P. P., \& Silva, A. S. (2019). Morte Encefálica: Conhecimento e Opinião dos Médicos da Unidade de Terapia Intensiva. Revista Brasileira de Educação Médica, 43(3), 115-122. https://doi.org/10.1590/1981-52712015v43n3rb20180122

Souza, S. S., Borenstein, M. S., Silva, D. M. G. V., Souza, S. S., \& Carvalho, J. B. (2013a). Situações de estresse vivenciadas pela equipe de enfermagem no cuidado ao potencial doador de órgãos. Rev. pesqui. Cuid.fundam. (Online), 42-52. https://pesquisa.bvsalud.org/enfermeria/resource/pt/lil-683548

Souza, S. S., Borenstein, M. S., Silva, D. M. G. V., Souza, S. S., \& Carvalho, J. B. (2013b). Estratégias de enfrentamento da enfermagem no cuidado ao potencial doador de órgãos. Rev Rene (Online), 92-100. https://doi.org/10.15253/2175-6783.2013000100011

Vesco, N. L., Nogueira, C. S., Lima, R. F., Souza, V. N., Brasil, B. M. B. L., \& Viana, C. D. M. R. (2016). Conhecimento do enfermeiro na manutenção do potencial doador de órgãos e tecidos para transplante. Rev. enferm. UFPE on line, 10(5), 1615-1624. Retrieved from http://www.revista.ufpe.br/revistaenfermagem/index.php/revista/article/viewFile/8986/pdf_10146

Westphal, G. A., Garcia, V. D., Souza, R. L. de, Franke, C. A., Vieira, K. D., Birckholz, V. R. Z., ... Andrade, J. de. (2016). Guidelines for the assessment and acceptance of potential brain-dead organ donors. Revista Brasileira de Terapia Intensiva, 28(3), 220-255. https://doi.org/10.5935/0103-507X.20160049 